\title{
COUNTING PREIMAGES
}

\author{
MICHAŁ MISIUREWICZ AND ANA RODRIGUES
}

\begin{abstract}
For noninvertible maps, mainly subshifts of finite type and piecewise monotone interval maps, we investigate what happens if we follow backward trajectories, random in the sense that at each step every preimage can be chosen with equal probability. In particular, we ask what happens if we try to compute the entropy this way. It tuns out that instead of the topological entropy we get the metric entropy of a special measure, which we call the fair measure. In general this entropy (the fair entropy) is smaller than the topological entropy. In such a way, for the systems that we consider, we get a new natural measure and a new invariant of topological conjugacy.
\end{abstract}

\section{InTRODUCTION, MOTIVATION AND QUESTIONS}

When working on topological entropy of one-dimensional dynamical systems, one would like to have some simple method of computing it. The simplest class of such systems is the class of piecewise strictly monotone interval maps. Various computational methods are known (see, e.g., $[5,6,7]$ ), but none of them is really general and simple. Even for the Markov maps, before starting computations (which are relatively simple, because one computes only the spectral radius of a non-negative matrix, see, e.g., [1]) one has to identify the Markov structure and find the transition matrix. This structure may be complicated and the matrix can be large.

One of the obvious ideas for computing topological entropy is to count preimages of a given point.

Definition 1.1. The class $\mathcal{P} \mathcal{M M}$ (piecewise monotone mixing) consists of all continuous maps $f:[0,1] \rightarrow[0,1]$ which are piecewise monotone (with finitely many pieces) and topologically mixing.

Clearly, if a map $f$ is in $\mathcal{P} \mathcal{M M}$ then it is piecewise strictly monotone. It is also known (see, e.g., [4]) that it is locally eventually onto, that is, for every nonempty open set $U$ there is $n$ such that $f^{n}(U)=[0,1]$.

We can compute the topological entropy $h(f)$ of $f \in \mathcal{P} \mathcal{M M}$ by counting preimages of any point. This follows immediately from the well known properties of interval maps.

Theorem 1.2. If $f \in \mathcal{P} \mathcal{M M}$ and $x \in[0,1]$ then

$$
h(f)=\lim _{n \rightarrow \infty} \frac{1}{n} \log \operatorname{Card}\left(f^{-n}(x)\right) .
$$

Date: August 29, 2015.

2010 Mathematics Subject Classification. 37E05, 37A35, 37B10. 
Proof. If $c_{n}$ is the number of laps (pieces of monotonicity) of $f^{n}$, then

$$
h(f)=\lim _{n \rightarrow \infty} \frac{1}{n} \log c_{n}
$$

(see, e.g., [1]). Since in each lap of $f^{n}$ there can be at most one element of $f^{-n}(x)$, we get

$$
h(f) \geq \limsup _{n \rightarrow \infty} \frac{1}{n} \log \operatorname{Card}\left(f^{-n}(x)\right) .
$$

On the other hand, if $f^{k}$ has an $s$-horseshoe, that is, there are intervals $K_{1}, \ldots, K_{s}$ with pairwise disjoint interiors such that $\bigcup_{j=1}^{s} K_{j} \subset f^{k}\left(K_{i}\right)$ for each $i$, then for every $y \in K_{1}$ we have $\operatorname{Card}\left(f^{-k}(y)\right) \geq s / 2$, so by induction, $\operatorname{Card}\left(f^{-k m}(y)\right) \geq(s / 2)^{m}$ for $m=1,2, \ldots$ The map $f^{k}$ is a surjection, so $\operatorname{Card}\left(f^{-k m-r}(y)\right) \geq(s / 2)^{m}$ for $r=0,1, \ldots, k-1$. Therefore $\operatorname{Card}\left(f^{-n}(y)\right) \geq(s / 2)^{n / k-1}$ for $n=1,2, \ldots$ Since $f$ is locally eventually onto, there exists $\ell$ and $y \in K_{1}$ such that $f^{\ell}(y)=x$. This gives us $\operatorname{Card}\left(f^{-n}(x)\right) \geq(s / 2)^{(n-\ell) / k-1}$ for $n=1,2, \ldots$, and thus

$$
\liminf _{n \rightarrow \infty} \frac{1}{n} \log \operatorname{Card}\left(f^{-n}(x)\right) \geq \frac{1}{k} \log (s / 2)=\frac{1}{k} \log s-\frac{1}{k} \log 2 .
$$

There are increasing sequences $\left(k_{i}\right)$ and $\left(s_{i}\right)$ such that $f^{k_{i}}$ has an $s_{i}$-horseshoe and

$$
h(f)=\lim _{i \rightarrow \infty} \frac{1}{k_{i}} \log s_{i}
$$

(see, e.g., [1]), and therefore by (4) we get

$$
\liminf _{n \rightarrow \infty} \frac{1}{n} \log \operatorname{Card}\left(f^{-n}(x)\right) \geq h(f) .
$$

Together with (3) this proves (1).

While this is an interesting theoretical result, it is usually useless if we want to compute topological entropy using a computer. The number of preimages of $x$ under $f^{n}$ grows exponentially with $n$, and keeping track of all of them requires a lot of memory, while computing their preimages (when we pass from $n$ to $n+1$ ) requires a lot of time.

In some similar situations, the problem is solved by replacing the full tree of preimages by one branch, chosen randomly. This type of procedure is used for instance to draw an attractor for an Iterated Function System (see, e.g., [3]) or the Julia set for a rational map of the Riemann sphere to itself (see, e.g., [8]). In fact, in the latter case the measures equidistributed along longer and longer pieces of a random backward trajectory converge in the weak-* topology to the measure with maximal entropy (see [8]).

Thus, our procedure will be the following. Denote by $c(x)$ the cardinality of the set $f^{-1}(x)$. We start with a point $x_{0}$ and proceed by induction. Given $x_{n}$ we choose $x_{n+1}$ from the set $f^{-1}\left(x_{n}\right)$ randomly, that is, the probability of choosing any of those points is $1 / c\left(x_{n}\right)$. Then we go to the limit with the geometric averages of $c\left(x_{0}\right), c\left(x_{1}\right), \ldots, c\left(x_{n}\right)$ as $n$ goes to infinity. We hope that the limit exists and its logarithm is the topological entropy of $f$. Moreover, we hope that the measures equidistributed along longer and longer pieces of a random backward trajectory converge in the weak-* topology to the measure with maximal entropy. 
Alas, a simple computer experiment where $f$ is a unimodal map with the turning point periodic of period 3 suggests that the limit of geometric averages exists, but is approximately 1.5874 instead of the expected golden ratio (that is, approximately 1.618). This immediately modifies our expectations, by removing reference to the topological entropy from them. However, we want to know what is going on.

Question 1.3. What can we say about the convergence of the geometric averages of $c\left(x_{0}\right), c\left(x_{1}\right), \ldots, c\left(x_{n}\right)$ as $n$ goes to infinity for a random choice of the backward trajectory? Does it exist for every initial point $x_{0}$ ? For almost every initial point in some sense? How much does the limit depend on the initial point?

Question 1.4. What can we say about the convergence of the measures equidistributed along longer and longer pieces of a random backward trajectory? Does it exist for every initial point $x_{0}$ ? For almost every initial point in some sense? How much does the limit depend on the initial point?

Question 1.5. What is the connection between the limits mentioned in the two preceding questions? In particular, is the logarithm of the first limit the entropy of the measure that is the second limit?

Of course the questions can be asked not only for interval maps. We will give full answers for transitive subshifts of finite type and almost full answers for interval maps from the class $\mathcal{P} \mathcal{M} \mathcal{M}$. Let us also mention that the questions are meaningful only for noninvertible systems.

In general, we expect to get a measure and its entropy, and we hope they will be unique. Then we can speak of the fair measure and the fair entropy. Clearly, if two systems are conjugate, the conjugacy will carry the fair measure to the fair measure, so in particular, the fair entropies of those systems will be equal. In such a way we get an invariant of topological conjugacy.

Let us note a big difference between the answers to our questions that apply to every point and to almost every point. W really aim at the "every point" theorems. "Almost every" would mean practically "almost every with respect to the fair measure." However, we do not know a priori the fair measure, so if the system has some natural measure, this may mean "almost none with respect to the natural measure." Fortunately, in the cases that we consider, even if we cannot get the "every" results, we will be able to get something more than "almost every."

The paper is organized as follows. In Section 2 we provide the definitions and describe what can be done without too many conditions on the dynamical system. This is continued in Section 3, where we consider the natural extension of the system. In Sections 4 and 5 we investigate closer the special classes of maps: subshifts of finite type and piecewise monotone topologically mixing interval maps, respectively. We obtain for those classes stronger results than in the general case. In Section 6 we show how the results for interval maps can be strengthened by looking at the preimage trees. In Section 7 we show that the strongest results that we obtained for subshifts of finite type apply also for the mixing Markov interval maps. In Section 8 we find necessary and sufficient conditions for the fair entropy to be equal to the topological entropy for subshifts of finite type. Finally, in Section 9 we show that for unimodal maps the assumption of transitivity can be skipped. 


\section{General case}

While in the preceding section we were talking mainly about single backward trajectories, when we want to prove something about them, we have to have information about the whole trees of preimages. Therefore we will be working in the following setup.

Let $X$ be a compact metric space and $f: X \rightarrow X$ a continuous map. We assume that $f$ is a surjection, and that there exists a partition $\mathcal{X}$ of $X$ into Borel sets $X_{i}$, $i=1,2, \ldots s$, such that for every $i$ the restriction of $f$ to $X_{i}$ is a homeomorphism onto its image (which is also a Borel set).

We define a partition $\mathcal{A}$ as the common refinement of the partitions $\left\{f\left(X_{i}\right), X \backslash\right.$ $\left.f\left(X_{i}\right)\right\}, i=1,2, \ldots s$. Clearly, $\mathcal{A}$ is a finite partition of $X$ into Borel sets. For each $A \in \mathcal{A}$ denote by $p(A)$ the set of those numbers $i \in\{1,2, \ldots s\}$ for which $A \subset f\left(X_{i}\right)$. Thus, each $x \in A$ has a preimage in every $X_{i}$ such that $i \in p(A)$ and no more preimages. In particular, the number $c(x)$ of preimages of $x$ depends only on $A$ (and therefore can be denoted $c(A))$. Since $f$ is a surjection, this number is always positive.

Let $\mathfrak{M}$ be the space of all probability Borel measures on $X$. We will define an operator $\Phi$ from $\mathfrak{M}$ to itself. If $\mu \in \mathfrak{M}$, then we chop $\mu$ into pieces $\left.\mu\right|_{A}, A \in \mathcal{A}$, divide each piece by $c(A)$ and push via $\left(\left.f\right|_{X_{i}}\right)^{-1}$ to each $X_{i}$ with $i \in p(A)$. That is, we set

$$
\Phi(\mu)=\sum_{A \in \mathcal{A}} \sum_{i \in p(A)}\left(\left.f\right|_{X_{i}}\right)_{*}^{-1}\left(\frac{\left.\mu\right|_{A}}{c(A)}\right) .
$$

In other words, if $B \subset X$ is a Borel set then

$$
\begin{aligned}
\Phi(\mu)(B)=\sum_{A \in \mathcal{A}} \sum_{i \in p(A)} \frac{\mu\left(f\left(X_{i} \cap B\right) \cap A\right)}{c(A)} \\
\quad=\sum_{A \in \mathcal{A}} \sum_{i=1}^{s} \frac{\mu\left(f\left(X_{i} \cap B\right) \cap A\right)}{c(A)}=\sum_{i=1}^{s} \sum_{A \in \mathcal{A}} \frac{\mu\left(f\left(X_{i} \cap B\right) \cap A\right)}{c(A)} .
\end{aligned}
$$

In particular, if $B \subset X_{i}$ and $f(B) \subset A \in \mathcal{A}$, then $\mu(f(B))=\Phi(\mu)(B) \cdot c(A)$.

Observe that if we replace the partition $\mathcal{A}$ by a finer one, the operator $\Phi$ will not change. Therefore this operator does not depend on a particular choice of the partition $\mathcal{X}$, as long as this partition satisfies the assumptions we made. Indeed, for two different partitions we can take their common refinement, and that will lead to the common refinement of the corresponding partitions $\mathcal{A}$.

The operator $\Phi$ is a partial inverse of the operator $f_{*}$ that carries the measure forward. Namely, it follows immediately from the definition of $\Phi$ that

$$
f_{*} \circ \Phi=\mathrm{id} .
$$

We will be looking for fixed points of $\Phi$. If $\mu$ is a fixed point of $\Phi$ then for a set $B$ contained in an element $A$ of $\mathcal{A}$ its preimage consists of $c(A)$ sets $B_{j}$, each of them contained in one of the sets $X_{j}$, and $\mu\left(B_{j}\right)=\mu(B) / c(A)$ for every $j$. Thus, if we go backward, the chance of choosing any of $j$ s is the same. In other words, our procedure for choosing a preimage is fair. This motivates the following name.

Definition 2.1. A measure $\mu \in \mathfrak{M}$ will be called a fair measure if $\Phi(\mu)=\mu$. 
From the definition of $\Phi$ and the definition of the measure-theoretic Jacobian (see, e.g., [13]) we get immediately the following lemma.

Lemma 2.2. A measure $\mu \in \mathfrak{M}$ is fair if and only if its measure-theoretic Jacobian is $x \mapsto c(f(x))$.

Another point of view on the Jacobian is to speak about the $g$-measures (see, e.g., $[10,11,14])$. The condition that the Jacobian of $\mu$ is $x \mapsto c(f(x))$ is equivalent to $\mu$ being a $g$-measure, where $g$ is the reciprocal of the Jacobian. Therefore another way of stating Lemma 2.2 is as follows.

Lemma 2.3. A measure $\mu \in \mathfrak{M}$ is fair if and only if it is a $g$-measure for the function $g$ given by $g(x)=1 / c(f(x))$.

Continuing the translation into the language of $g$-measures, our operator $\Phi$ is dual to the Ruelle operator for the function $\xi=\log g$, that is, the function given by $\xi(x)=$ $-\log c(f(x))$. However, for interval maps this operator is usually not continuous. Moreover, the function $\xi$ usually does not satisfy the inequality $\sup \xi-\inf \xi<h_{\text {top }}(f)$, often assumed when the properties of $g$-measures are considered (see, e.g., [9]).

We will later need the following lemma.

Lemma 2.4. If a measure $\mu \in \mathfrak{M}$ is fair, so is its almost every ergodic component.

Proof. Let $\mu \in \mathfrak{M}$ be fair. Ergodic components of $\mu$ are conditional measures $\mu_{y}$ for a measurable partition $\eta$ into invariant subsets, and there is the corresponding measure $\varkappa$ on the factor space $Y$, such that for every measurable set $B \subset X$

$$
\mu(B)=\int_{Y} \mu_{y}(B) d \varkappa(y) .
$$

By Lemma 2.2, the Jacobian of $\mu$ is $x \mapsto c(f(x))$. This must be also the Jacobian of almost every ergodic component of $\mu$. Indeed, if this is not the case, then there is a measurable set $A \subset X$ with $\mu(A)>0$, on which the Jacobian of every ergodic component of $\mu$ is larger than the Jacobian of $\mu$ (or maybe we have to replace "larger" by "smaller", but the proof stays the same). We may assume that $f$ is one-to-one on $A$. However, then (with the natural notation for Jacobians)

$$
\begin{aligned}
\mu(f(A))=\int_{Y} \mu_{y}(f(A)) & d \varkappa(y)=\int_{Y}\left(\int_{A} J_{y}(z) d \mu_{y}(z)\right) d \varkappa(y) \\
> & \int_{Y}\left(\int_{A} J(z) d \mu_{y}(z)\right) d \varkappa(y)=\int_{A} J(z) d \mu(z)=\mu(f(A)),
\end{aligned}
$$

a contradiction. Thus, again by Lemma 2.2, almost every ergodic component of $\mu$ is fair.

The natural procedure for finding a fixed point of $\Phi$ would be to start with an arbitrary measure $\nu \in \mathfrak{M}$, consider the sequence of averages

$$
\left(\frac{1}{n} \sum_{i=0}^{n-1} \Phi^{i}(\nu)\right)_{n=1}^{\infty}
$$

and take a weak-* limit of a subsequence of this sequence. The problem is that, in general, the operator $\Phi$ is not continuous. Therefore there is no guarantee that this 


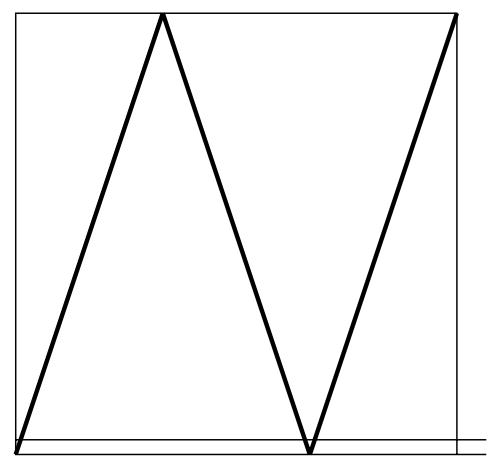

Figure 1. An example where $\Phi$ is discontinuous.

limit will be a fixed point of $\Phi$. Even for piecewise monotone interval maps one can easily see that $\Phi$ can be discontinuous.

Example 2.5. Consider a full 3-horseshoe $f:[0,1] \rightarrow[0,1]$, that is, the "connect the dots" map with "dots" given by $f(0)=0, f(1 / 3)=1, f(2 / 3)=0$ and $f(1)=1$ (see Figure 1). If $\delta_{x}$ is the Dirac delta measure at $x$ then $\Phi\left(\delta_{0}\right)=\left(\delta_{0}+\delta_{2 / 3}\right) / 2$. However, if instead of 0 we take a point $\varepsilon$ close to 0 , we get $\Phi\left(\delta_{\varepsilon}\right)=\left(\delta_{\varepsilon / 3}+\delta_{(2-\varepsilon) / 3}+\delta_{(2+\varepsilon) / 3}\right) / 3$. While $\delta_{\varepsilon} \rightarrow \delta_{0}$ as $\varepsilon \rightarrow 0$, the measures $\Phi\left(\delta_{\varepsilon}\right)$ converge to $\left(\delta_{0}+2 \delta_{2 / 3}\right) / 3$ instead of $\Phi\left(\delta_{0}\right)$.

Nevertheless, we can say something about the accumulation points of the sequence (7).

Lemma 2.6. Any accumulation point of the sequence (7) is an $f$-invariant measure. Proof. Let $\left(n_{j}\right)_{j=1}^{\infty}$ be an increasing sequence of positive integers and assume that

$$
\lim _{j \rightarrow \infty} \frac{1}{n_{j}} \sum_{i=0}^{n_{j}-1} \Phi^{i}(\nu)=\mu
$$

in the weak-* topology. If $\varphi: X \rightarrow \mathbb{R}$ is a continuous function, then by (6) we have

$$
\begin{aligned}
&\left|f_{*}\left(\frac{1}{n_{j}} \sum_{i=0}^{n_{j}-1} \Phi^{i}(\nu)\right)(\varphi)-\left(\frac{1}{n_{j}} \sum_{i=0}^{n_{j}-1} \Phi^{i}(\nu)\right)(\varphi)\right| \\
&=\frac{1}{n_{j}}\left|\left(f_{*}(\nu)+\sum_{i=0}^{n_{j}-2} \Phi^{i}(\nu)\right)(\varphi)-\left(\sum_{i=0}^{n_{j}-1} \Phi^{i}(\nu)\right)(\varphi)\right| \\
&=\frac{1}{n_{j}}\left|\left(f_{*}(\nu)(\varphi)-\Phi^{n_{j}-1}(\nu)\right)(\varphi)\right| \leq \frac{2\|\varphi\|}{n_{j}}
\end{aligned}
$$

and this converges to 0 as $j \rightarrow \infty$. Therefore, by the continuity of $f_{*}$, we get $f_{*}(\mu)=\mu$. This means that $\mu$ is $f$-invariant.

Similarly, we get the following result. 
Lemma 2.7. Set

$$
\nu_{n}=\frac{1}{n} \sum_{k=0}^{n-1} \Phi^{k}(\nu)
$$

and let $\left(n_{j}\right)_{j=1}^{\infty}$ be an increasing sequence of positive integers such that $\lim _{j \rightarrow \infty} \nu_{n_{j}}=\mu$ in the weak-* topology. Then also $\lim _{j \rightarrow \infty} \Phi\left(\nu_{n_{j}}\right)=\mu$ in the weak-* topology.

Proof. If $\varphi: X \rightarrow \mathbb{R}$ is a continuous function, then we have

$$
\begin{aligned}
\left|\Phi\left(\nu_{n_{j}}\right)(\varphi)-\nu_{n_{j}}(\varphi)\right|=\frac{1}{n_{j}} \mid\left(\sum_{i=1}^{n_{j}} \Phi^{i}(\nu)\right)(\varphi)- & \left(\sum_{i=0}^{n_{j}-1} \Phi^{i}(\nu)\right)(\varphi) \mid \\
& =\frac{1}{n_{j}}\left|\Phi^{n_{j}}(\nu)(\varphi)-\nu(\varphi)\right| \leq \frac{2\|\varphi\|}{n_{j}}
\end{aligned}
$$

and this converges to 0 as $j \rightarrow \infty$. Therefore, if the sequence $\left(\nu_{n_{j}}\right)_{j=1}^{\infty}$ converges, then the sequence $\left(\Phi\left(\nu_{n_{j}}\right)\right)_{j=1}^{\infty}$ converges to the same limit.

\section{NATURAL EXTENSION}

Let us try to make connections between various approaches that we employed. Suppose that we constructed a fair measure $\mu$. Consider the natural extension $(\hat{X}, \hat{f}, \hat{\mu})$ of the system $(X, f, \mu)$, and let $\pi: \hat{X} \rightarrow X$ be the natural projection. Then the partition of $\hat{X}$ into preimages of points under $\pi$ is a measurable partition. Therefore there is a canonical system of conditional measures $\left(\mu_{x}\right)_{x \in X}$, where $\mu_{x}$ is a probability measure in $\pi^{-1}(x)$. Since $\pi_{*}(\hat{\mu})=\mu$, this means that for every measurable set $B \subset \hat{X}$ we have

$$
\hat{\mu}(B)=\int_{X} \mu_{x}\left(B \cap \pi^{-1}(x)\right) d \mu(x) .
$$

If $x \in X$ then $\pi^{-1}(x)$ the set of (infinite) branches of the preimage tree rooted at $x$. To build this tree we start at $x$ and draw edges from $x$ to its preimages under $f$. Then we continue by induction: from every vertex $y$ we draw edges to the elements of $f^{-1}(y)$. Since we want to get a tree, if $x$ is periodic then the same point of $X$ can represent countably many vertices (so formally a vertex is of the form $(y, n)$, where $f^{n}(y)=x$; this vertex is in the $n$th generation of vertices).

In the space $\pi^{-1}(x)$ we can also consider a probability measure $\nu_{x}$ given by the Jacobian of $\mu$. To define this measure it is enough to specify the measures of sets $[y, n]_{x}$, where $[y, n]_{x}$ is the set of all branches of the tree that pass through the vertex $y$ of the $n$th generation. If $J$ is the measure-theoretic Jacobian of $f$ for the measure $\mu$, then we set

$$
\nu_{x}\left([y, n]_{x}\right)=\frac{1}{J(y) \cdot J(f(y)) \cdot \ldots \cdot J\left(f^{n-1}(y)\right)}=\frac{1}{c(f(y)) \cdot c\left(f^{2}(y)\right) \cdot \ldots \cdot c\left(f^{n}(y)\right)} .
$$

Clearly, this choice of measures is consistent, that is,

$$
\sum_{z \in f^{-1}(y)} \nu_{x}\left([z, n+1]_{x}\right)=\nu_{x}\left([y, n]_{x}\right) .
$$

Therefore the definition of $\nu_{x}$ is correct. 
The measures $\nu_{x}$ are exactly the measures that we use our "random choice" of a backward trajectory. Note that their definition is really independent of the measure $\mu$, because the function $x \mapsto c(f(x))$ does not depend on $\mu$. Instead of the natural extension, we can use the inverse limit in the sense of topological dynamics and we get the same $\hat{X}, \hat{f}$ and $\pi$. Thus, even if we do not know anything about the existence of a fair measure (like in our questions), we can use the following terminology.

Definition 3.1. We will say that some condition is satisfied for a random backward trajectory of $x \in X$ if it is satisfied for $\nu_{x}$ almost every element of $\pi^{-1}(x)$.

The following lemma provides a link between our questions and fair measures.

Lemma 3.2. If $\mu$ is a fair measure then $\mu_{x}=\nu_{x}$ for almost every $x$.

Proof. We have to check that (9) is satisfied if we replace $\mu_{x}$ with $\nu_{x}$. Every measurable subset of $\hat{X}$ can be approximated (in the sense of measure $\hat{\mu}$ ) by finite unions of the sets $B$ of the following form. There exists a measurable set $C \subset X$ and $A_{1}, A_{2}, \ldots, A_{n} \in \mathcal{A}$ such that $f^{i}(C) \subset A_{i}$ for $i=1,2, \ldots, n$, and

$$
B=\left\{\left(\ldots, x_{2}, x_{1}, x_{0}\right): x_{n} \in C\right\} .
$$

Thus, we can assume that the set $B$ in (9) is of such form. Then

$$
\nu_{x}\left(B \cap \pi^{-1}(x)\right)=\frac{1}{c\left(A_{1}\right) \cdot c\left(A_{2}\right) \cdot \ldots \cdot c\left(A_{n}\right)}
$$

if $x \in f^{n}(C)$ and $\nu_{x}\left(B \cap \pi^{-1}(x)\right)=0$ otherwise. Thus,

$$
\int_{X} \nu_{x}\left(B \cap \pi^{-1}(x)\right) d \mu(x)=\frac{\mu\left(f^{n}(C)\right)}{c(f(y)) \cdot c\left(f^{2}(y)\right) \cdot \ldots \cdot c\left(f^{n}(y)\right)}=\mu(C)=\hat{\mu}(B) .
$$

This proves that $\nu_{x}$ satisfies (9), and therefore $\nu_{x}=\mu_{x}$ almost everywhere.

Remark 3.3. With more work we can remove from Lemma 3.2 the assumption that the measure $\mu$ is fair, when we define $\nu_{x}$ only using the Jacobian of $\mu$ (without the numbers $\left.c\left(x_{i}\right)\right)$. However, we do not need here such general result.

Now, if $\mu$ is a fair ergodic measure, we can get a partial answer to our questions.

Theorem 3.4. Let $\mu$ be a fair ergodic measure. Then:

(a) For $\mu$-almost every point $x_{0} \in X$, for a random choice of the backward trajectory of $x_{0}$ the geometric averages of $c\left(x_{0}\right), c\left(x_{1}\right), \ldots, c\left(x_{n}\right)$ converge to $\exp \left(\int \log c(x) d \mu(x)\right)$ as $n$ goes to infinity.

(b) For $\mu$ almost every point $x_{0} \in X$, for a random choice of the backward trajectory of $x_{0}$ the measures $(1 / n) \sum_{k=0}^{n-1} \delta_{x_{k}}$ converge in the weak-* topology to $\mu$.

(c) If additionally $f$ has a one-sided generator, then the limit in (a) is the exponential of the entropy of $\mu$.

Proof. Use the Birkhoff Ergodic Theorem for the system $\left(\hat{X}, \hat{f}^{-1}, \hat{\mu}\right)$ and the function $x \mapsto \log c(\pi(x))$. The phrase "for $\hat{\mu}$-almost every $y \in \hat{X}$ " means the same as "for $\mu$-almost every $x \in X$ and $\mu_{x}$-almost every $y \in \pi^{-1}(x)$ ". Thus, by Lemma 3.2 and in view of Definition 3.1, we get convergence of averages of $\log c(x)$ to

$$
\int \log c(\pi(y)) d \hat{\mu}(y)=\int \log c(x) d \mu(x) .
$$


This proves (a).

If additionally $f$ a one-sided generator, then the entropy is the integral of the logarithm of the Jacobian, so $h_{\mu}(f)=\int \log c(f(x)) d \mu(x)=\int \log c(x) d \mu(x)$ (the second equality holds because $\mu$ is invariant), and this proves (c).

Finally, the proof of (b) is almost the same as the proof of (a), except that instead of the ergodic theorem we use the fact that almost every point of $\hat{X}$ is generic for $\hat{\mu}$.

Of course, since we get the answers not for every point of $X$, but only for almost every one (with respect to some special measure), those are not full answers. Moreover, they are answers only when we know that a fair measure exists and is ergodic. In fact, we would like a unique fair measure, because otherwise the limits can heavily depend on the point (one limit for almost every point for one measure, another limit for almost every point for another measure). We will address the existence and uniqueness of the fair measure in some cases in the next sections.

\section{Subshifts of Finite TYPE}

Suppose that $(X, f)$ is a transitive one-sided subshift of finite type with the $0-1$ transition matrix $M=\left(m_{i j}\right)$. Then $c(x)$ depends only on the cylinder of length 1 to which $x$ belongs, and if it is the $j$ th cylinder then it is equal to

$$
c_{j}=\sum_{i} m_{i j}
$$

We can choose as $X_{i}$ the $i$ th cylinder of length 1 , and $f\left(X_{i}\right)$ is a union of finitely many cylinders of length 1 . Therefore both the sets $X_{i}$ and the elements of the partition $\mathcal{A}$ are clopen (close and open) sets. This implies that the operator $\Phi$ is continuous.

In this situation we can use for instance the results of [11] to deduce the existence and uniqueness of the fair measure, as well as its strong ergodic properties. Alternatively, we can use the method mentioned in Section 2. However, we can do better, namely we can construct a fair measure explicitly. This way we get also explicit formula for its entropy, which is the limit that we are looking for.

Write

$$
q_{i j}=\frac{m_{i j}}{c_{j}}
$$

and consider the matrix $Q=\left(q_{i j}\right)$. We have

$$
\sum_{i} q_{i j}=\frac{1}{c_{j}} \sum_{i} m_{i j}=\frac{c_{j}}{c_{j}}=1 .
$$

This means that the vector with all components 1 is a left eigenvector of $Q$ corresponding to the eigenvalue 1 . This implies that the spectral radius of $Q$ is 1 , so there is a unique probability vector $p=\left(p_{i}\right)$ which is a right eigenvector of $Q$ corresponding to the eigenvalue 1 . Thus,

$$
\sum_{j} q_{i j} p_{j}=p_{i} \quad \text { and } \quad \sum_{i} p_{i}=1 .
$$

Moreover, each $p_{i}$ is strictly positive. 
Now we set

We have

$$
p_{i j}=\frac{q_{i j} p_{j}}{p_{i}}=\frac{m_{i j} p_{j}}{c_{j} p_{i}}
$$

and

$$
\sum_{j} p_{i j}=\sum_{j} \frac{q_{i j} p_{j}}{p_{i}}=\frac{p_{i}}{p_{i}}=1
$$

$$
\sum_{i} p_{i} p_{i j}=\sum_{i} q_{i j} p_{j}=p_{j}
$$

Therefore there is a Markov measure $\mu$ with probabilities of the states $p_{i}$ and transition probabilities $p_{i j}$. We have $\mu(X)=1$, because if $m_{i j}=0$ then $p_{i j}=0$.

The measure-theoretic Jacobian $J$ of $\mu$ is constant on cylinders of length 2 , and on the $i j$ th cylinder it is equal to

$$
\frac{p_{j}}{p_{i} p_{i j}}=\frac{p_{j}}{m_{i j} p_{j} / c_{j}}=c_{j}
$$

provided this cylinder is nonempty, that is, $m_{i j}=1$. Thus, by Lemma 2.2 , the measure $\mu$ is fair.

Whenever $m_{i j}>0$, we have also $p_{i j}>0$. Thus, since our subshift of finite type was transitive, we see that $\mu$ is ergodic. Therefore we can use Theorem 3.4. Moreover, in our specific situation we can strengthen it.

Theorem 4.1. Let $(X, f)$ be a transitive subshift of finite type. Then for the fair measure $\mu$ constructed above:

(a) For every point $x_{0} \in X$, for a random choice of the backward trajectory of $x_{0}$ the geometric averages of $c\left(x_{0}\right), c\left(x_{1}\right), \ldots, c\left(x_{n}\right)$ converge to $\exp \left(h_{\mu}(f)\right)$ as $n$ goes to infinity.

(b) For every point $x_{0} \in X$, for a random choice of the backward trajectory of $x_{0}$ the measures $(1 / n) \sum_{k=0}^{n-1} \delta_{x_{k}}$ converge in the weak-* topology to $\mu$.

Moreover, $\mu$ is the only fair measure for $f$.

Proof. If the 0th coordinate of the points $x_{0}$ and $y_{0}$ are equal then the backward trees for those points look the same, that is, there is a natural bijection between them. This bijection sends the measure $\nu_{x_{0}}$ to $\nu_{y_{0}}$ and if the branch $\left(y_{n}\right)$ corresponds to $\left(x_{n}\right)$ then the distance between $x_{n}$ and $y_{n}$ goes to 0 as $n \rightarrow \infty$. Therefore, if the choice of the backward trajectory of $x_{0}$ gives the convergence described in Theorem 3.4 (a) and (b), then the choice of the corresponding backward trajectory of $y_{0}$ gives the same convergence. This means that this convergence occurs for every $y_{0}$. Moreover, in our case $\mathcal{X}$ is a generator, so we can use Theorem 3.4 (c). This completes the proof of (a) and (b).

If there is another fair measure for $f$, then by Lemma 2.4 there is another ergodic fair measure, and by Theorem 3.4 this contradicts (b).

Now we can look back at the results of the computer experiment described in Section 1. The interval map considered there was Markov with the $2 \times 2$ transition matrix $\left(m_{i j}\right)$, where $m_{12}=m_{21}=m_{22}=1$ and $m_{11}=0$. Therefore $c_{1}=1$ and $c_{2}=2$. This gives us

$$
Q=\left[\begin{array}{ll}
0 & 1 / 2 \\
1 & 1 / 2
\end{array}\right],
$$


and thus $p_{1}=1 / 3$ and $p_{2}=2 / 3$. Hence, $p_{11}=0, p_{12}=1$ and $p_{21}=p_{22}=1 / 2$. Now we can compute the entropy of $\mu$ :

$$
h_{\mu}(f)=-\sum_{i, j} p_{i} p_{i j} \log p_{i j}=-\frac{2}{3} \log \frac{1}{2}=\log \left(2^{2 / 3}\right) \approx \log 1.5784 .
$$

Thus, the number that we got in the experiment was (as it should be) the exponential of the entropy of the unique fair measure for the corresponding subshift of finite type. We will elaborate on the connection between the interval Markov maps and subshifts of finite type in Section 7.

\section{Piecewise monotone interval maps}

We will show that in spite of the discontinuity of $\Phi$, the procedure mentioned in Section 2 works for piecewise monotone interval maps.

Thus, in this section our space is $X=[0,1]$, a map $f: X \rightarrow X$ is continuous, piecewise monotone and topologically mixing (that is, it belongs to $\mathcal{P} \mathcal{M M}$ ), and $X_{i}$, $i=1,2, \ldots, s$, are intervals of monotonicity of $f$. We really want $\mathcal{X}$ to be a partition, so we assign each turning point of $f$ to only one of the intervals $X_{i}, X_{i+1}$. Note that then some elements of the partition $\mathcal{A}$ are singletons consisting of the image of a turning point.

Lemma 5.1. Let $K$ be a subinterval of $X$ and let $\varkappa \in \mathfrak{M}$. Then

$$
\Phi(\varkappa)(K) \geq \frac{1}{s} \varkappa(f(K))
$$

Proof. We can partition $K$ into subintervals (some of them perhaps degenerate) by intersecting with the preimages of the elements of $\mathcal{A}$. For each of those subintervals (11) holds (with this subinterval replacing $K$ ), by the definition of $\Phi$. Taking the sum of those inequalities, we get (11) for $K$. In fact, at the right-hand side we may get even more than $(1 / s) \varkappa(f(K))$, because the images of our subintervals need not be disjoint, but the inequality goes in the right direction.

Lemma 5.2. Let $\mu \in \mathfrak{M}$ be an accumulation point of the sequence (7) and let $K$ be a subinterval of $X$ such that $\mu$-measures of the boundary of $K$ and of the boundary of $f(K)$ are zero. Then

$$
\mu(K) \geq \frac{1}{s} \mu(f(K)) .
$$

Proof. Define $\nu_{n}$ as in (8) and assume that $\left(n_{j}\right)_{j=1}^{\infty}$ is an increasing sequence of positive integers such that $\lim _{j \rightarrow \infty} \nu_{n_{j}}=\mu$ in the weak-* topology. Let us recall that the weak-* convergence of measures implies convergence of measures of sets whose boundaries have limit measure zero. Therefore, by Lemmas 2.7 and 5.1 we get

$$
\mu(K)=\lim _{j \rightarrow \infty} \Phi\left(\nu_{n_{j}}\right)(K) \geq \frac{1}{s} \lim _{j \rightarrow \infty} \nu_{n_{j}}(f(K))=\frac{1}{s} \mu(f(K)) .
$$

Lemma 5.3. Let $\mu \in \mathfrak{M}$ be an accumulation point of the sequence (7). Then $\mu$ is nonatomic. 
Proof. Suppose that $\mu$ has an atom at some point $x \in X$. Since $\mu$ is invariant by Lemma 2.6, $\mu\left(\left\{f^{n}(x)\right\}\right) \geq \mu(\{x\})$ for every $n>0$. Therefore $x$ has to be periodic and if $P$ is the periodic orbit of $x$ then $\mu(\{y\})=\mu(\{x\})$ for every $y \in P$. It follows that if $f^{k}(z)=x$ and $z \notin P$ then $\mu(\{z\})=0$.

As we mentioned, $f$ is topologically exact (locally eventually onto). Thus, there exists an interval $\tilde{L} \subset X$ disjoint from $P$ and a number $k>0$ such that $f^{k}(\tilde{L})=$ $X$. Let $K$ be an interval containing $x$, whose boundary has measure $\mu$ zero. By Lemma 1.2.1 of [1], there is a subinterval $\hat{L} \subset \tilde{L}$ such that $f^{k}(\hat{L})=K$. Applying this lemma once more, we get a subinterval $L \subset \hat{L}$ such that $f^{k}(L)=K$ and the endpoints of $L$ are mapped to the endpoints of $K$. Since the boundary of $K$ has measure $\mu$ zero, and $\mu$ is invariant, we see that the boundary of $f^{i}(L)$ for $i=0,1, \ldots, k-1$ has also measure $\mu$ zero.

A minor problem can occur because in Lemma 5.2 the boundary is understood as the boundary relative to the interval $X$, so an endpoint of $K$ may not belong to the boundary of $K$ if it is an endpoint of $X$. However, then we can extend slightly $L$ so that its image stays the same, but the singleton of the image under $f^{k}$ of the corresponding endpoint of $L$ has measure zero. This cannot be done if the endpoint of $L$ where we want to enlarge $L$ is an endpoint of $X$, but then this endpoint does not belong to the boundary of $L$, so the problem disappears.

As the result, we get a situation where we can apply Lemma $5.2 k$ times. Taking a descending sequence of intervals $K_{n}$ with intersection $\{x\}$ we get by induction a descending sequence of intervals $L_{n}$. By Lemma 5.2, $\mu\left(L_{n}\right) \geq\left(1 / s^{k}\right) \mu\left(K_{n}\right) \geq \mu(\{x\})$ for every $n$. The intersection of the intervals $L_{n}$ cannot be a nondegenerate interval because its image under $f^{k}$ is $\{x\}$ and $f$ is mixing. Thus, it is a singleton of some point $z$. Since $L$ was disjoint from $P$, we have $z \notin P$. We have $\mu(\{z\})>0$, which contradicts what we proved earlier.

We say that a measure has full support if every open nonempty set has positive measure.

Lemma 5.4. Let $\mu \in \mathfrak{M}$ be an accumulation point of the sequence (7). Then $\mu$ has full support.

Proof. By Lemmas 5.2 and 5.3, the inequality (12) holds for every interval $K$. If $K$ is a nondegenerate interval, then there is $s$ such that $f^{s}(K)=X$. Then, by (12), $\mu(K)>0$.

Now we are ready to prove the existence of a fair measure.

Theorem 5.5. Every accumulation point of the sequence (7) is a fair measure.

Proof. Let $\mu \in \mathfrak{M}$ be an accumulation point of the sequence (7). We will show that if $K$ is a subinterval of $X$ such that $f(K)$ is contained in some element $A \in \mathcal{A}$, then

$$
\mu(K)=\frac{1}{c(A)} \mu(f(K)) .
$$

This means that the Jacobian of $\mu$ is $x \mapsto c(f(x))$, so by Lemma $2.2 \mu$ is fair.

Define $\nu_{n}$ as in (8) and assume that $\left(n_{j}\right)_{j=1}^{\infty}$ is an increasing sequence of positive integers such that $\lim _{j \rightarrow \infty} \nu_{n_{j}}=\mu$ in the weak-* topology. By the definition of $\Phi$, we 
have

$$
\Phi\left(\nu_{n}\right)(K)=\frac{1}{c(A)} \nu_{n}(f(K))
$$

for every $n$. By Lemma 5.3, both $K$ and $f(K)$ have boundaries of measure $\mu$ zero. Therefore, by Lemma 2.7, we get

$$
\mu(K)=\lim _{j \rightarrow \infty} \Phi\left(\nu_{n_{j}}\right)(K)=\frac{1}{c(A)} \lim _{j \rightarrow \infty} \nu_{n_{j}}(f(K))=\frac{1}{c(A)} \mu(f(K)) .
$$

Remark 5.6. If $\mu$ is a fair measure, then we can use $\mu$ as the starting measure $\nu$. Then the sequence ( 7 ) is constant, so $\mu$ satisfies all lemmas about the accumulations points of this sequence. In particular, $\mu$ is $f$-invariant, nonatomic, and has full support.

Now we prove uniqueness of the fair measure. We start with a lemma which is probably well known, but it is simpler to prove it than to find it in the literature.

Lemma 5.7. Let $\mu$ and $\nu$ be mutually singular nonatomic probability measures with full support on $X$. Then for $\mu$-almost every $x \in X$

$$
\lim _{\varepsilon, \delta \rightarrow 0} \frac{\nu([x-\delta, x+\varepsilon])}{\mu([x-\delta, x+\varepsilon])}=0 .
$$

Proof. Since the measure $\mu+\nu$ is nonatomic and has full support, we may assume that it is the Lebesgue measure (we apply a homeomorphism of $X$ that carries it to the Lebesgue measure). Since $\mu$ and $\nu$ are mutually singular, there is a Borel set $A \subset X$ with $\mu(A)=1$ and $\nu(A)=0$. By the (one-sided) Lebesgue Density Theorem, for $\mu$-almost every $x \in A$ (and therefore for $\mu$-almost every $x \in X$ ) we have

$$
\lim _{\varepsilon, \delta \rightarrow 0} \frac{\mu([x-\delta, x+\varepsilon])}{\delta+\varepsilon}=\lim _{\varepsilon, \delta \rightarrow 0} \frac{(\mu+\nu)([x-\delta, x+\varepsilon] \cap A)}{\delta+\varepsilon}=1 .
$$

Since

$$
\delta+\varepsilon=(\mu+\nu)([x-\delta, x+\varepsilon])=\mu([x-\delta, x+\varepsilon])+\nu([x-\delta, x+\varepsilon]),
$$

we get from $(15)$

$$
\lim _{\varepsilon, \delta \rightarrow 0} \frac{\nu([x-\delta, x+\varepsilon])}{\delta+\varepsilon}=0 .
$$

Now (14) follows from (15) and (16).

The above lemma shows that in a small scale the mutually singular measures are very incompatible. Now we show that in a large scale the situation is quite different.

Lemma 5.8. Let $\mu$ and $\nu$ be nonatomic probability measures with full support on $X$. Then for each $\varepsilon>0$ there exists $\delta>0$ such that if $K \subset X$ is an interval and $\mu(K) \geq \varepsilon$ then $\nu(K) \geq \delta$.

Proof. Suppose that his is not true. Then there exists a sequence of intervals $\left[a_{n}, b_{n}\right] \subset$ $X$ such that $\mu\left(\left[a_{n}, b_{n}\right]\right) \geq \varepsilon$ and $\nu\left(\left[a_{n}, b_{n}\right]\right)<1 / n$. By compactness, we may assume that $a_{n} \rightarrow a$ and $b_{n} \rightarrow b$ as $n \rightarrow \infty$. Since $\mu$ is nonatomic, we have $\mu([a, b]) \geq \varepsilon$, and therefore $b>a$. Similarly, we get $\nu([a, b])=0$. Since $\nu$ has full support, this implies that $b=a$ and we get a contradiction. This concludes the proof. 
Finally, we have to have tools to pass from a small to a large scale. The following lemma is well known, and is basically a special case of Lemma 4.1 of [12]. Notice that although formally in [12] the assumption is that the slope is constant, what is really used in the proof is only that it is larger than 2. Note also that in our terminology "piecewise" means piecewise with finitely many pieces.

Lemma 5.9. Let $g: X \rightarrow X$ be a piecewise linear map with slopes on each piece larger than 2. Then there exists $\varepsilon>0$ such that for Lebesgue almost every $x \in X$ there exists an increasing sequence $\left(n_{k}\right)$ of positive integers such that for every $k$ there exists an interval $K_{k}$ containing $x$ such that $g^{n_{k}}$ is linear on $K_{k}$ and the length of $g^{n_{k}}\left(K_{k}\right)$ is at least $\varepsilon$.

Now we are prepared to prove the result which is the main step for proving uniqueness of the fair measure.

Proposition 5.10. Let $\mu, \nu \in \mathfrak{M}$ be fair measures. Then they cannot be mutually singular.

Proof. Since $f$ is mixing, there is $n$ such that $f^{n}$ is at least 2-to- 1 except perhaps one point. Since by Remark $5.6 \mu$ is nonatomic and has full support, we may assume that it is the Lebesgue measure. Then the slope of $f^{n}$ on each lap is at least 2 , so the slope of $f^{2 n}$ on each slope is at least 4. By Lemma 5.9 there is $\varepsilon$ such that for $\mu$-almost every $x \in X$ there exists an increasing sequence $\left(n_{k}\right)$ of positive integers such that for every $k$ there exists an interval $K_{k}$ containing $x$ such that $f^{n n_{k}}$ is linear on $K_{k}$ and the length of $f^{n n_{k}}\left(K_{k}\right)$ is at least $\varepsilon$. By Lemma 5.8 there is $\delta>0$ (depending only on $\varepsilon)$ such that $\nu\left(f^{n n_{k}}\left(K_{k}\right)\right) \geq \delta$. Since $\mu$ and $\nu$ are fixed points of $\Phi$, we have

$$
\frac{\nu\left(K_{k}\right)}{\mu\left(K_{k}\right)}=\frac{\nu\left(f^{n n_{k}}\left(K_{k}\right)\right)}{\mu\left(f^{n n_{k}}\left(K_{k}\right)\right)} \geq \delta .
$$

Thus, by Lemma 5.7, $\mu$ and $\nu$ cannot be mutually singular.

Now we are ready to prove uniqueness of the fair measure.

Theorem 5.11. The map $f$ has only one fair measure.

Proof. If there are two distinct fair measures, by Lemma 2.4 there are two distinct ergodic fair measures. Then they are mutually singular, which contradicts Proposition 5.10 .

Now let us look at the broader picture. We can start with any measure $\nu \in \mathfrak{M}$, take an accumulation point of the sequence (7) and we always get the same fair measure. We will denote this measure by $\mu_{\text {fair }}$.

Theorem 5.12. For every $\nu \in \mathfrak{M}$ the sequence (7) converges in the weak-* topology to $\mu_{\text {fair }}$.

Proof. Since the space $\mathfrak{M}$ is compact, every subsequence of (7) has a convergent subsequence, and by Theorems 5.5 and 5.11, the limit is $\mu_{\text {fair }}$. Therefore the whole sequence (7) converges to $\mu_{\text {fair }}$.

Let us look at the entropy of the fair measure for the tent maps and compare it with the topological entropy (see Figure 2). The maps are parametrized by the slope, so the graph of the exponential of the topological entropy is a straight line. Except for 


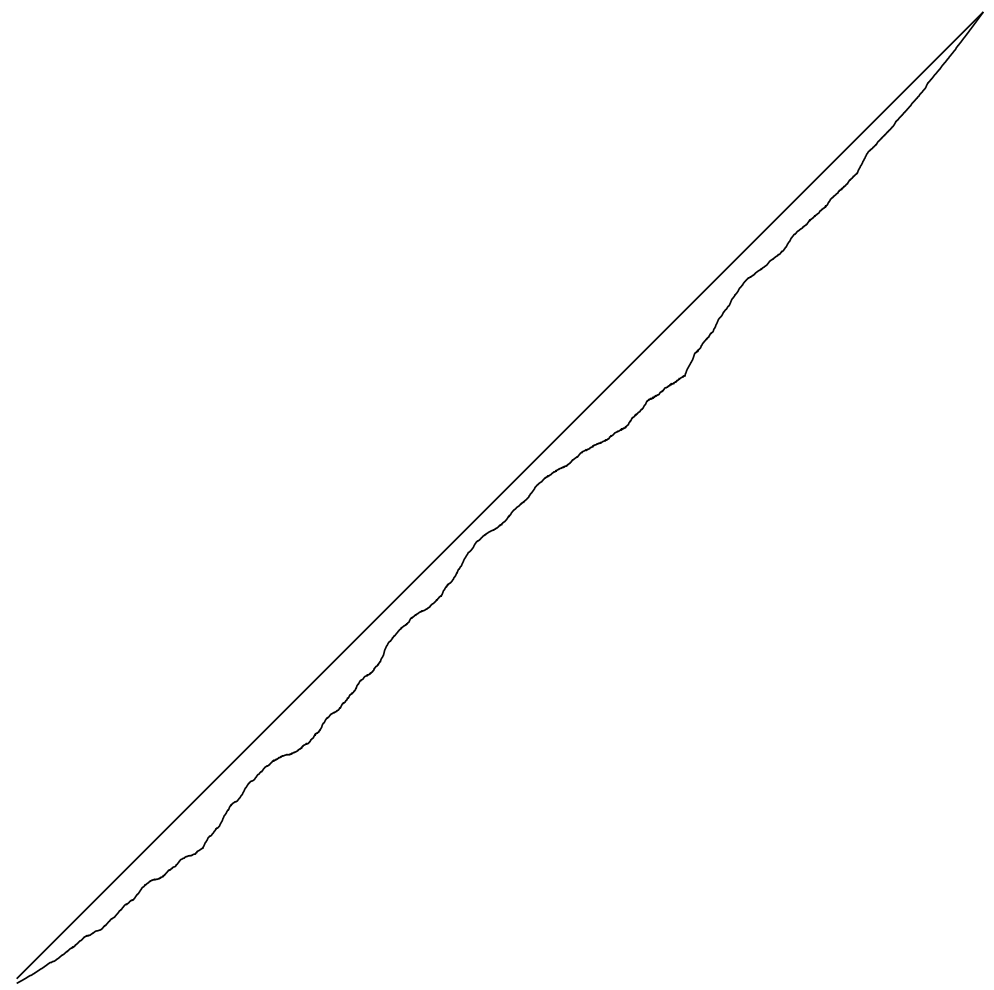

Figure 2. The graphs of $\exp \left(h_{\mu_{\text {fair }}}(f)\right)$ and $\exp \left(h_{\text {top }}(f)\right)$ for tent maps $f$ with slopes from 1.42 to 2 .

the slope 2 (and the slope $\sqrt{2}$, which is just to the left of the beginning of the graphs; the map is transitive but not mixing then) the entropy of the fair measure is smaller than the topological entropy. However, the difference is not large. The entropy of the fair measure as the function of the slope looks continuous and increasing. We will prove that in fact this is the case.

Theorem 5.13. For the family of the tent maps, parametrized by the slope from $(\sqrt{2}, 2]$, the entropy of the fair measure is continuous and strictly increasing as the function of the parameter.

Proof. Denote by $f_{a}$ the tent map with the slope $a$, and by $g_{b}$ the unimodal map defined as the "connect the dots" map on $[0,1]$ with the dots $(0,1-b),(b / 2,1)$, $(b, 1-b)$ and $(1,0)$. Then the Jacobian of $g_{b}$ for the Lebesgue measure is 2 on $[0, b]$ and 1 on $(b, 1]$, while $g_{b}$ is 2-to- 1 on $g_{b}([0, b])$ and 1-to- 1 on $g_{b}((b, 1])$ (see Figure 3$)$. Therefore the Lebesgue measure is the fair measure. Its entropy is the integral of the logarithm of the Jacobian so it is equal to $b \log 2$. If we allow also $a=\sqrt{2}$ and $b=1 / 2$ then, since each $g_{b}$ is transitive, there exists exactly one $a=\xi(b)$ for which $g_{b}$ is conjugate to $f_{a}$ (see, e.g., [2]). The topological entropy is a continuous function of a unimodal map with positive entropy (see [1]), so $\xi$ is a continuous function. All maps $g_{b}$ for $b \in(1 / 2,1]$ are topologically mixing, so $\xi(b) \in(\sqrt{2}, 2]$. Moreover, $\xi(1 / 2)=\sqrt{2}$ and $\xi(1)=2$. If $\xi$ is not strictly increasing then there exist two maps $g_{b}$ and $g_{b^{\prime}}$ which are conjugate to the same $f_{a}$, so they are conjugate to each other. 

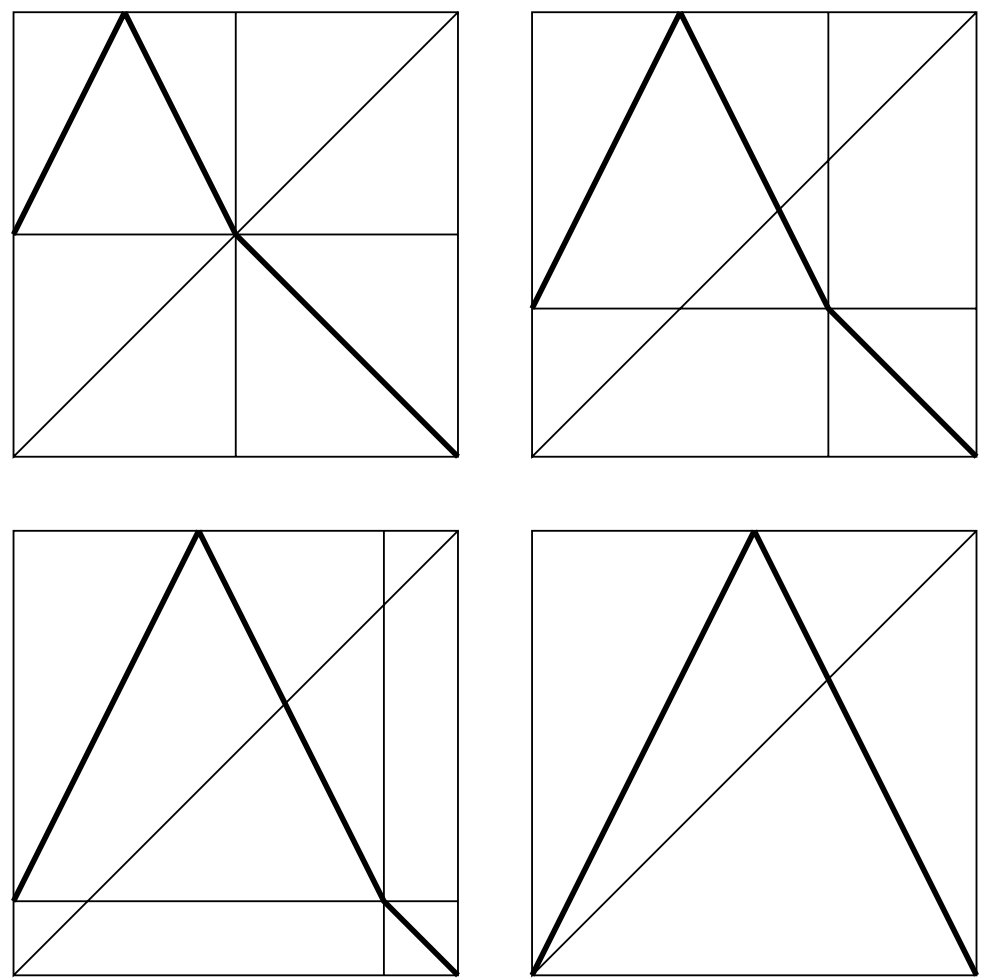

Figure 3. The maps $g_{b}$.

This contradicts the uniqueness of the fair measure. Thus, $\xi$ is a homeomorphism, so $\xi^{-1}$ is well defined, continuous and strictly increasing.

\section{Preimage tree}

In Section 3 for $x \in X$ we considered the preimage tree rooted at $x$ with the measure $\nu_{x}$ on the space $Y_{x}$ of the branches of this tree. Let us take a closer look at this object and see what we can say when $f \in \mathcal{P} \mathcal{M M}$.

Let our initial measure be $\delta_{x}$. By Theorem 5.12, the sequence

$$
\left(\frac{1}{n} \sum_{i=0}^{n-1} \Phi^{i}\left(\delta_{x}\right)\right)_{n=1}^{\infty}
$$

converges to the fair measure $\mu$ in the weak-* topology. This means that if $\varphi: X \rightarrow \mathbb{R}$ is a continuous function then the sequence

$$
\left(\frac{1}{n} \sum_{i=0}^{n-1} \int \varphi d \Phi^{i}\left(\delta_{x}\right)\right)_{n=1}^{\infty}
$$

converges to $\int \varphi d \mu$.

For every vertex $y$ of $n$th generation in the tree we have the measure

$$
m(y, n)=\nu_{x}\left([y, n]_{x}\right)=\frac{1}{c(f(y)) \cdot c\left(f^{2}(y)\right) \cdot \ldots \cdot c\left(f^{n}(y)\right)}
$$



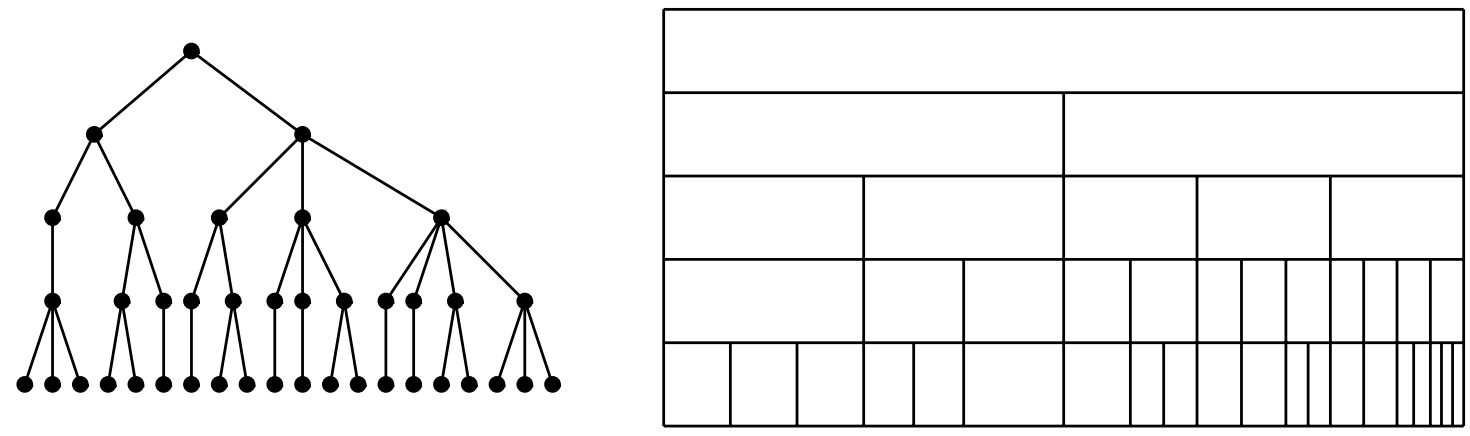

FiguRE 4. Five first rows of a tree and its rectangular model.

assigned to it. Moreover, from the definition of $\Phi$ we get by induction

$$
\Phi^{i}\left(\delta_{x}\right)=\sum_{y \in f^{-i}(x)} m(y, i) \delta_{y} .
$$

Therefore, (17) can be rewritten as

$$
\left(\frac{1}{n} \sum_{i=0}^{n-1} \sum_{y \in f^{-i}(x)} m(y, i) \varphi(y)\right)_{n=1}^{\infty}
$$

Now it will be beneficial to change the way we look at the tree. We replace each vertex of the tree by a rectangle. Thus, the vertex $y$ of the $n$th generation will become a rectangle in the $n$th row, with the width $m(y, n)$. Above it are its ancestors and below it its descendants (see Figure 4). Remember that really the whole picture goes down to infinity. In this model the branches are vertical half-lines starting at the top of the rectangle and going down. The measure $\nu_{x}$ corresponds to the Lebesgue measure on the horizontal interval. We ignore the vertical lines containing the partition lines, because there are only countably many of them, so this is a set of measure zero.

Now we can interpret the inner sum in (18) as follows. Define functions $\varphi_{i}: Y_{x} \rightarrow \mathbb{R}$ by setting $\varphi_{i}(z)$ equal to the value of $\varphi$ at the point of $i$ th generation on the branch $z$. In our rectangular model, this means that in each rectangle we write the value of $\varphi$ at the corresponding vertex of the tree, and $\varphi_{i}(z)$ is the value we see in the rectangle at the level $i$ at the intersection with the vertical line corresponding to $z$. Now we see clearly that

$$
\sum_{y \in f^{-i}(x)} m(y, i) \varphi(y)=\int_{Y_{x}} \varphi_{i}(z) d \nu_{x}(z),
$$

so the convergence of (18) to $\int \varphi d \mu$ can be written as

$$
\lim _{n \rightarrow \infty} \frac{1}{n} \sum_{i=0}^{n-1} \int_{Y_{x}} \varphi_{i}(z) d \nu_{x}(z)=\int \varphi d \mu .
$$

Observe now that if $y \in f^{-k}(x)$ then the tree rooted at $y$ is a subtree of the tree rooted at $x$. In the rectangular model, we have to take the (infinite) subrectangle consisting of the rectangle corresponding to $y$ in the $k$ th generation and all rectangles 
below it. In the horizontal direction this means that we take a subinterval. The measure $\nu_{y}$ is the Lebesgue measure on this subinterval, normalized. If we replace $x$ by $y$, Theorem 5.12 still holds, and this gives us the formula analogous to (19). When we go back to the notation of (19), we use the fact that for the convergence of averages first $k$ terms do not matter; integration over $Y_{x}$ (the whole horizontal interval) should be replaced by the integration over the subinterval, and the right-hand side should be multiplied by the measure of this subinterval (because we normalized the measure). That is, we get the formula

$$
\lim _{n \rightarrow \infty} \frac{1}{n} \sum_{i=0}^{n-1} \int_{B} \varphi_{i}(z) d \nu_{x}(z)=\nu_{x}(B) \int \varphi d \mu .
$$

This formula holds for all sets $B$ of the form $[y, k]_{x}$. However, the finite unions of such sets approximate all Borel subsets of $Y_{x}$, and the functions $\varphi_{i}$ are commonly bounded, so we get the following lemma.

Lemma 6.1. Equation (20) holds for all Borel subsets $B \subset Y_{x}$.

In such a way we arrived to the following model. We have a sequence of commonly bounded measurable function $\psi_{n}:[0,1] \rightarrow \mathbb{R}$ (they correspond to the averages of functions $\varphi_{i}$ minus $\int \varphi d \mu$ ), such that for every measurable set $A \subset[0,1]$ we have

$$
\lim _{n \rightarrow \infty} \int_{A} \psi_{n}(x) d x=0 .
$$

We would like to deduce that $\lim _{n \rightarrow \infty} \psi_{n}(x)=0$ for almost every $x \in[0,1]$.

Unfortunately, this is not the case. Let $\psi_{n}(x)$ be equal to 1 if the $n$th digit of the binary expansion of $x$ is 1 , and -1 otherwise. Then the integrals of $\psi_{n}$ over any measurable set $A$ go to 0 , but the sequence $\left(\psi_{n}\right)_{n=1}^{\infty}$ diverges almost everywhere.

Therefore we have to look for a weaker result.

Lemma 6.2. In the situation described above, for every $x \in[0,1]$ the sequence $\left(\psi_{n}(x)\right)_{n=1}^{\infty}$ either diverges or converges to 0 .

Proof. If there is a set of positive measure on which the limit exists but is not 0 , then there is a constant $c>0$ and a set of positive measure $A \subset[0,1]$ such that for every $x \in A$ we have $\lim _{n \rightarrow \infty} \psi_{n}(x) \geq c$ (or for every $x \in A$ this limit is less than or equal to $-c$, in which case the proof is the same). Since the functions $\psi_{n}$ are commonly bounded, we get

$$
\lim _{n \rightarrow \infty} \int_{A} \psi_{n}(x) d x \geq c
$$

a contradiction.

Now, translating this result to our real situation, we get immediately the following proposition.

Proposition 6.3. If $f: X \rightarrow X$ belongs to $\mathcal{P} \mathcal{M M}$ and $x_{0} \in X$ then for every continuous function $\varphi: X \rightarrow \mathbb{R}$ and for a random choice of the trajectory of $x_{0}$ the sequence

$$
\left(\frac{1}{n} \sum_{k=0}^{n-1} \varphi\left(x_{k}\right)\right)_{n=1}^{\infty}
$$


either diverges or converges to $\int \varphi d \mu$.

As a corollary, we get the following theorem.

Theorem 6.4. Let $f: X \rightarrow X$ belong to $\mathcal{P} \mathcal{M M}$ and let $\mu$ be the fair measure. Then:

(a) For every point $x_{0} \in X$, for a random choice of the backward trajectory of $x_{0}$ the geometric averages of $c\left(x_{0}\right), c\left(x_{1}\right), \ldots, c\left(x_{n}\right)$ either converge to $\exp \left(h_{\mu}(f)\right)$ or diverge.

(b) For every point $x_{0} \in X$, for a random choice of the backward trajectory of $x_{0}$ the measures $(1 / n) \sum_{k=0}^{n-1} \delta_{x_{k}}$ either converge in the weak-* topology to $\mu$ or diverge.

Proof. To prove (a) just apply Proposition 6.3 to the function $\varphi(x)=\log c(x)$ and use Theorem 3.4 (c).

To prove (b), apply Proposition 6.3 to the functions from a countable basis of the space of the continuous real-valued functions on $X$.

\section{INTERVAL MARKOV MAPS}

In this section we show that while for general mixing piecewise monotone interval maps the answers to our questions are not full, the situation is better for Markov maps, where we can use the results of Section 4.

Thus, let $X=[0,1]$ and let the map $f: X \rightarrow X$ be continuous, piecewise monotone, Markov, and topologically mixing.

Theorem 7.1. In the above situation there is a unique fair measure for $f$, and:

(a) For every point $x_{0} \in X$, for a random choice of the backward trajectory of $x_{0}$ the geometric averages of $c\left(x_{0}\right), c\left(x_{1}\right), \ldots, c\left(x_{n}\right)$ converge to $\exp \left(h_{\mu}(f)\right)$ as $n$ goes to infinity.

(b) For every point $x_{0} \in X$, for a random choice of the backward trajectory of $x_{0}$ the measures $(1 / n) \sum_{k=0}^{n-1} \delta_{x_{k}}$ converge in the weak-* topology to $\mu$.

Proof. The uniqueness of the fair measure follows from Theorem 5.11.

Since $f$ is Markov, there is a corresponding subshift of finite type $g: Y \rightarrow Y$. The only obstruction to the systems $(X, f)$ and $(Y, g)$ being conjugate is that the intervals of the Markov partition are closed, so the endpoints of those intervals different than 0 and 1 belong to two intervals (so, strictly speaking, it is not a partition).

Let $P$ be the set of endpoints of the intervals of the Markov partition. There are only countably many points whose forward or backward trajectories pass through $P$, and the fair measures for $f$ and $g$ are nonatomic, so those systems with their fair measures are isomorphic. Moreover, if the preimage tree of a point $x_{0} \in X$ does not contain a point of $P$ then its preimage tree is the same (including the measure) as the preimage tree of its counterpart for $g$, and (a) and (b) follow from Theorem 4.1.

Thus, we can restrict our attention to the points whose backward trees pass through $P$. Since $P$ is invariant, it follows that the root $x_{0}$ of such a tree belongs to $P$. Except for the branches that pass through $P$ infinitely often, the rest of the tree after removing the vertices from $P$ splits into countably many subtrees rooted at a vertex not belonging to $P$. For those subtrees (a) and (b) hold, and, as we already noticed in Section 6 this implies that for the corresponding parts of the tree rooted at $x_{0}$ also (a) 
and (b) hold. If a branch passes through $P$ infinitely often, then, since $P$ is invariant and finite, it is periodic. Therefore there are only finitely many such branches, and since we are talking about a random choice of the backward trajectory, we can ignore them (they constitute a set of measure zero for the measure $\nu_{x_{0}}$ ). This completes the proof.

\section{Maximal ENTROpy}

Let us recall that our motivation was to compute the topological entropy of a map. As we saw, we get instead the fair entropy. Thus, we can ask when fair entropy and the topological entropy coincide. Let us consider this question for topologically mixing subshifts of finite type.

Thus, let $f: X \rightarrow X$ be a topologically mixing subshift of finite type with the transition matrix $M=\left(m_{i j}\right)$. Then it has the unique fair measure $\mu_{\text {fair }}$ and the unique measure of maximal entropy $\mu_{\max }$.

The spectral radius of $M$ is $\lambda$, where $\log \lambda$ is the topological entropy of $f$. For the eigenvalue $\lambda, M$ has positive eigenvectors: a left one $\left(\ell_{i}\right)$ and a right one $\left(r_{i}\right)$. According to the Parry's formulas, $\mu_{\max }$ is a Markov measure with measures of 1-cylinders $[i]$ equal to $\ell_{i} r_{i}$ (where we normalize eigenvectors in such a way that $\sum_{i} \ell_{i} r_{i}=1$ ), and the transition probability from $i$ to $j$ is

$$
\frac{m_{i j} r_{j}}{\lambda r_{i}}
$$

Lemma 8.1. If $m_{i j}=1$ then the Jacobian of $\mu_{\max }$ on the 2 -cylinder $[i j]$ is $\lambda \ell_{j} / \ell_{i}$.

Proof. The Jacobian on this cylinder is equal to the measure of the 1-cylinder $[j]$ divided by the measure of the 2-cylinder $[i j]$. Thus, it is

$$
\frac{\ell_{j} r_{j}}{\ell_{i} r_{i} \frac{m_{i j} r_{j}}{\lambda r_{i}}}=\frac{\ell_{j} r_{j} \lambda r_{i}}{\ell_{i} r_{i} m_{i j} r_{j}}=\frac{\lambda \ell_{j}}{\ell_{i}}
$$

To state a theorem, we need some definitions. We say that functions $\varphi, \psi: X \rightarrow \mathbb{R}$ are cohomologous if there is a function $v: X \rightarrow \mathbb{R}$ such that $\varphi-\psi=v \circ f-v$. In our case the functions $\varphi$ and $\psi$ will be constant on 1-cylinders, so in this definition we will assume that $u$ is also constant on 1-cylinders. Consider the case when the functions are $\varphi=\log c \circ f$ and $\psi$ is constant. Then we can write $\psi=\log K$, and in case when $\varphi$ and $\psi$ are homologous take $u=e^{v}$. Then $\varphi$ and $\psi$ are homologous if and only if there exists a vector $\left(u_{i}\right)$ such that if $m_{i j}=1$ then

$$
\log c_{j}-\log K=\log u_{j}-\log u_{i}
$$

that is,

$$
u_{i}=\frac{K u_{j}}{c_{j}}
$$

We will use the standard representation of our subshift of finite type by a graph. If $L$ is a loop in this graph, then we will denote by $C(L)$ the product of the values of $c$ along this loop and by $\Lambda(L)$ the product of $\lambda$ s along the loop, that is, $\Lambda(L)=\lambda^{|L|}$, where $|L|$ is the length of $L$. We will call a loop simple if it passes through every vertex of the graph at most once. 
Now we are ready to state the theorem.

Theorem 8.2. Let $f$ be a topologically mixing subshift of finite type. With the notation explained above, the following conditions are equivalent:

(i) The fair entropy of $f$ is equal to its topological entropy.

(ii) The function $\log c \circ f$ is cohomologous to a constant.

(iii) $\mu_{\text {fair }}=\mu_{\max }$.

(iv) For every $i, j$ such that $m_{i j}=1$ we have $\ell_{i}=\lambda \ell_{j} / c_{j}$.

(v) For every loop $L$ in the graph of $f$ we have $C(L)=\Lambda(L)$.

(vi) For every simple loop $L$ in the graph of $f$ we have $C(L)=\Lambda(L)$.

Proof. (i) $\Rightarrow$ (iii) follows from the uniqueness of the measure of maximal entropy; (iii) $\Rightarrow$ (i) is immediate.

(iii) $\Leftrightarrow$ (iv) follows from Lemmas 8.1 and 2.2.

(iv) $\Rightarrow$ (ii) is immediate. To prove (ii) $\Rightarrow($ iv), for every $j$ use (21) and (10) to get

$$
\sum_{i} m_{i j} u_{i}=\frac{K u_{j}}{c_{j}} \sum_{i} m_{i j}=K u_{j}
$$

This means that the positive vector $\left(u_{i}\right)$ is a left eigenvector of $M$ for a positive eigenvalue $K$. Therefore $K=\lambda$ and $u_{i}=t \ell_{i}$ for some positive constant $t$. This proves (iv).

(iv) $\Rightarrow(\mathrm{v})$ follows by multiplication of $\ell_{i}=\lambda \ell_{j} / c_{j}$ along the loop. To prove $(\mathrm{v}) \Rightarrow(\mathrm{iv})$, set $k_{1}=\ell_{1}$ and define other $k_{i}$ by induction along the arrows in the graph of $f$ using the formula $k_{i}=\lambda k_{j} / c_{j}$ whenever $m_{i j}=1$. The assumption that $C(L)=\Lambda(L)$ for every loop $L$ guarantees that this definition is consistent: when we get back to $i$ for which $k_{i}$ is already defined, we get the same value of $k_{i}$. Now for every $j$

$$
\sum_{i} k_{i} m_{i j}=\frac{\lambda k_{j}}{c_{j}} m_{i j}=\lambda k_{j}
$$

so $\left(k_{i}\right)$ is a left eigenvector of $M$ for the eigenvalue $\lambda$. Since $k_{1}=\ell_{1}$, this vector is equal to $\left(\ell_{i}\right)$. This proves (iv).

$(\mathrm{v}) \Rightarrow(\mathrm{vi})$ is immediate; (vi) $\Rightarrow(\mathrm{v})$ follows from the fact that every loop is a concatenation of simple loops.

Let us comment on the conditions in Theorem 8.2. We want to characterize mixing subshifts of finite type for which (i) (or (iii)) is true. From the theoretical point of view, (ii) is the main characterization. However, from the practical point of view, (vi) is the most important, because it is the simplest condition to check. Conditions (iv) and (v) are more technical and are included to make the proofs simpler.

The experiment illustrated in Figure 2 suggests that the fair entropy is equal to the topological entropy only if the function $c$ is constant. We will give an example of a mixing subshift of finite type when this is not true, and then explain why Figure 2 suggests the opposite. 


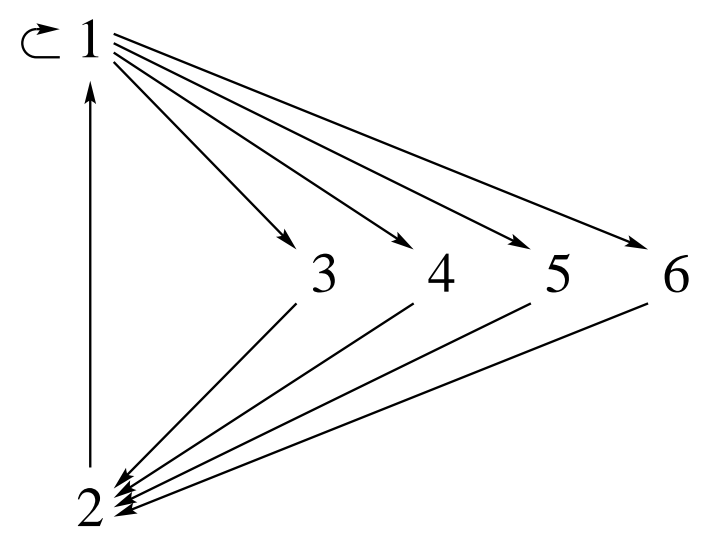

FiguRE 5. The graph of the subshift of finite type from the example.

Example 8.3. Let $f$ be the subshift of finite type with the graph as in Figure 5. That is, the transition matrix of $f$ is

$$
M=\left(\begin{array}{llllll}
1 & 0 & 1 & 1 & 1 & 1 \\
1 & 0 & 0 & 0 & 0 & 0 \\
0 & 1 & 0 & 0 & 0 & 0 \\
0 & 1 & 0 & 0 & 0 & 0 \\
0 & 1 & 0 & 0 & 0 & 0 \\
0 & 1 & 0 & 0 & 0 & 0
\end{array}\right)
$$

For every $n \geq 3$ there is a path from every vertex to every vertex in the graph, so $f$ is mixing. We have $c_{1}=2, c_{2}=4$, and $c_{i}=1$ for $i=3,4,5,6$. There are five simple loops: $1 \rightarrow 1$, and $1 \rightarrow i \rightarrow 2 \rightarrow 1$ for $i=3,4,5,6$. For the first simple loop, $L_{1}$, we have $C\left(L_{1}\right)=2$ and $\left|L_{1}\right|=1$. For any other simple loop $L$ we have $C(L)=8$ and $|L|=3$. The vector (2 $\left.2 \begin{array}{lllll}2 & 1 & 1 & 1 & 1\end{array}\right)$ is a left eigenvector of $M$ for the eigenvalue 2, so $\lambda=2$. Thus, condition (vi) of Theorem 8.2 is satisfied, so all other conditions of that theorem are also satisfied. Nevertheless, the function $c$ is not constant.

Now we look again at Figure 2 and try to explain it, at least for Markov maps.

Theorem 8.4. For Markov tent maps $f_{a}$ with slope $a \in(\sqrt{2}, 2]$ the fair entropy is equal to the topological entropy if and only if $a=2$.

Proof. For $a=2$ the Lebesgue measure is fair, and both the fair entropy and the topological entropy are equal to $\log 2$.

Assume now that $a<2$. The corresponding subshift of finite type $g: Y \rightarrow Y$ (as in the proof of Theorem 7.1) with the fair measure and with the measure of maximal entropy is isomorphic to $f_{a}$ with its fair measure and with its measure of maximal entropy, respectively, because all those measures are nonatomic. Therefore it is enough to prove that one of the conditions of Theorem 8.2 is violated for $g$.

The map $f_{a}$ has a fixed point $x$, and $x$ has two preimages. If $x$ belongs to the interior of one of the intervals of the Markov partition, then for the corresponding index $j$ for $g$ there is a loop $j \rightarrow j$ and $c_{j}=2$. Call this loop $L$. Then $C(L)=2$ and $\Lambda(L)=a$. This violates condition (vi). 
If $x$ does not belong to the interior of one of the intervals of the Markov partition, then it is a common endpoint of two of them, with indices $j$ and $k$. The map $f_{a}$ in a neighborhood of $x$ reverses orientation, so there is a loop $j \rightarrow k \rightarrow j$. Again, call this loop $L$. Then $C(L)=4$ and $\Lambda(L)=a^{2}$, and as before, this violates condition (vi). This completes the proof.

Conjecture 8.5. Theorem 8.4 holds for all tent maps $f_{a}$ with slope $a \in(\sqrt{2}, 2]$, not necessarily Markov.

While we do not know how to prove this conjecture, the numerical evidence (Figure 2) is strong. Moreover, the computations at the end of Section 4 together with the considerations in Section 7 show that when $a$ is the golden ratio then the fair entropy of $f_{a}$ is $\log 2^{2 / 3}$. Hence, by Theorem 5.13, if $2^{2 / 3} \leq a<(\sqrt{5}+1) / 2$ then the fair entropy of $f_{a}$ is less than $\log 2^{2 / 3}$. This proves that if $a \in\left[2^{2 / 3},(\sqrt{5}+1) / 2\right]$ then the fair entropy of $f_{a}$ is strictly smaller than its topological entropy.

\section{UNIMODAL MAPS}

Let us now consider the situation when $f: X \rightarrow X$ is a unimodal interval map. We assume that it is a surjection and that its entropy is larger than $\log \sqrt{2}$. However, we do not assume that it is mixing. Then $f$ is semiconjugate via a nondecreasing map $\pi$ to the tent map $g: Y \rightarrow Y$ with the slope $\exp (h(f))$, where $\mathrm{h}(\mathrm{f})$ is the topological entropy of $f$. Since $h(f)>\sqrt{2}$, the map $g$ is mixing, so we can use for it the results of Section 5, and if it is Markov, also Section 7.

To avoid misunderstandings, let us agree that when we say "unimodal," we mean that the map is increasing on the first lap and decreasing on the second one.

Compare the backward trajectories and the number of preimages for $f$ and $g$. When we pass via $\pi$, everything is the same, with two exceptions. Let $x$ be the left endpoint of $X$ and suppose that the point $x^{\prime}$ in the right lap of $f$ for which $f\left(x^{\prime}\right)=f(x)$ belongs to the interior of an interval $J \subset X$ which is mapped by $\pi$ to a point $y^{\prime} \in Y$. Then $g\left(y^{\prime}\right)$ has 2 preimages, while some points of $f(J)$ have only 1 preimage. The second exception is when an interval $K$ containing the turning point of $f$ is mapped by $\pi$ to a point. Then a point $x \in f(K)$ can have 2 preimages under $f$, while $\pi(x)$ has only 1 preimage under $g$.

However, in both cases, by the same reasons as in the proof of Theorem 7.1, for almost every choice of a backward trajectory of a given point, this trajectory passes through $J$ or $K$ only finitely many times. Therefore we may neglect those bad situations, and then $\pi$ establishes the correspondence between what happens for $f$ and $g$. Moreover, the fair measure for $f$ is well defined as the image under $\pi^{-1}$ of the fair measure for $g$, since the fair measure for $f$ is nonatomic.

Therefore, for any unimodal surjection $f$ with topological entropy larger than $\log \sqrt{2}$ there exists a unique fair measure, and Theorem 6.4 holds for $f$. Moreover, if the corresponding $g$ is Markov, a stronger Theorem 7.1 holds.

Note that if $f$ is renormalizable, then automatically $g$ is Markov.

Observe that the assumption $h(f)>\sqrt{2}$ is in a sense essential. Even for a tent map, if $h(f)<\sqrt{2}$ then there is an interval $I$ around the fixed point, such that every $x \in I$ has one preimage and $f^{-1}$ on $I$ is a contraction toward the fixed point. The union of images of $I$ under all iterates of $f$ is $X$, so for every $x \in X$ the random 
backward trajectory of $x$ sooner or later falls into $I$ and stays there. Therefore the unique fair measure is the Dirac delta at the fixed point and the fair entropy is 0 . That is, while a theorem analogous to Theorem 7.1 (without the assumption of Markov) holds, the situation is trivial, and far from satisfying. Moreover, Theorem 5.13 cannot be extended for parameters smaller than $\sqrt{2}$.

\section{REFERENCES}

[1] Ll. Alsedà, J. Llibre and M. Misiurewicz, "Combinatorial Dynamics and Entropy in Dimension One," Second Ed., Adv. Ser. Nonlinear Dynam. 5, World Sci., Singapore, 2000.

[2] Ll. Alsedà and M. Misiurewicz, Semiconjugacy to a map of a constant slope, to appear in Discrete Contin. Dyn. Syst. Ser. B.

[3] M. F. Barnsley, "Fractals Everywhere," Second Ed., Academic Press Professional, Boston, MA, 1993.

[4] L. Block and W. Coppel, "Dynamics in One Dimension," Lecture Notes in Math. 1513, Springer, Berlin, 1992.

[5] L. Block, J. Keesling,S. H. Li and K. Peterson, An improved algorithm for computing topological entropy, J. Statist. Phys. 55 (1989), 929-939.

[6] H. Bruin, An algorithm to compute the topological entropy of a unimodal map, Internat. J. Bifur. Chaos Appl. Sci. Engrg. 9 (1999), 1881-1882.

[7] R. Dilao and J. Amigo, Computing the topological entropy of unimodal maps, Internat. J. Bifur. Chaos Appl. Sci. Engrg. 22 (2012), 1250152, 14 pp.

[8] J. Hawkins and M. Taylor, Maximal entropy measure for rational maps and a random iteration algorithm, Internat. J. Bifur. Chaos Appl. Sci. Engrg. 13 (2003), 1442-1447.

[9] F. Hofbauer and G. Keller, Equilibrium states for piecewise monotonic transformations, Ergodic Theory Dynam. Systems 2 (1982), no. 1, 23-43.

[10] M. Keane, Strongly mixing g-measures, Invent. Math. 16 (1972), 309-324.

[11] F. Ledrappier, Principe variationnel et systmes dynamiques symboliques, Z. Wahrscheinlichkeitstheorie und Verw. Gebiete 30 (1974), 185-202.

[12] M. Misiurewicz and S. Roth, No semiconjugacy to a map of constant slope, Ergodic Theory Dynam. Systems (posted electronically on November 10, 2014).

[13] W. Parry, "Entropy and Generators in Ergodic Theory," W. A. Benjamin, Inc., New York, 1969.

[14] P. Walters, Ruelle's operator theorem and g-measures, Trans. Amer. Math. Soc. 214 (1975), 375-387.

Department of Mathematical Sciences, iUpUi, 402 N. Blackford Street, IndiANAPOLIS, IN 46202

E-mail address: mmisiure@math.iupui.edu

Mathematics, University of Exeter, Harrison Building, Streatham Campus, North

PARK RoAd, EXeter, UK, EX4 4QF

E-mail address: A.Rodrigues@exeter.ac.uk 